\title{
Ambient Assisted Living Monitoring System to Support Formal and Informal Caregivers for Elderly People
}

\author{
Francisco Javier Parada, Bruno Rosales, Wilhelm Stork \\ Embedded Systems and Sensors Engineering \\ FZI Research Center for Information Technologies \\ Karlsruhe, Germany \\ \{parada, rosales, stork\}@fzi.de
}

\begin{abstract}
Ambient Assisted Living (AAL) aims to provide services to support elderly people living more independently at their homes. Caregivers have an important role as they can monitor and support the persons, and evaluate if they can still live independently. Informal caregivers are important as well, as the demand of care is increasing while the number of healthcare professionals is decreasing and cannot cope with that demand. This paper proposes an AAL home monitoring system able to detect activities of daily living and more complex situations, like problematic behavioral changes, so it results in helpful information for both, formal and informal caregivers, in order to support them on their duties. The system is based on a context-aware middleware connected to sensors, and a web-based application for the monitoring. It will be evaluated for a long term in real world scenarios based on a user-oriented approach.
\end{abstract}

Keywords - AAL, Activity monitoring, Activity recognition, Telecare

\section{INTRODUCTION}

AAL is an emergent field with the background of the population ageing and it is growing very quickly. It aims to support elderly people to live longer and in a more autonomous way in their homes by means of ICT.

Although AAL focuses on elderly people, there are other groups who play an important role too, such as caregivers and healthcare professionals. Eldercare will also be affected by the ongoing demographic change: the decrease of active working population includes also professionals on the field; meanwhile the demand of services and care will increase together with the elderly population, leading to a rise, or even an overflowing, of the workload for caregivers. It is also worth mentioning the important role of the informal carers, such as family members or friends, who decide to directly care for the person. This is currently, for instance, the major group of caregivers in Germany, but the group is steadily diminishing, because of work incompatibility, distance from the person's home and because the increasing number of people who have no descendants [1]. Informal caregivers are confronted with new situations which are time and effort consuming, and, due to a lack of information and knowledge, this leads to very stressful predicaments and even becomes a burden on them [1]. Hence AAL focuses also on providing services and solutions to third persons involved in eldercare. Traditionally caregivers have used the everyday activities people perform (Activities of Daily Living, ADLs, see Table I) to evaluate their health status, mainly through assessment tools like Resident Assessment Instrument (RAI), Hamilton Depression Scale, Barthel Index, and so on. [2, 3, 4]. These evaluations imply a lot of paper work and, moreover, have the inconvenience of lack of reliability, as elderly people can always forget and/or omit relevant information.

In this work we propose an AAL home monitoring system for informal and formal caregivers. This system is based on situation recognition, aiming to detect not only ADLs, but relevant situations for the care monitoring of a person based on the ADLs, such as hygiene or nutrition problems, long inactivity, and cognitive problems.

\section{PROBLEM STATEMENT}

\section{A. Motivation}

To illustrate the problem and the possible solution we present the main Personas [5] in this work.

Martha is a 43-year-old woman married with two children. Her father is 70 and widowed for 5 years, and the doctors have diagnosed him a very initial stage of Alzheimer's disease. They have agreed that he continues living at home since he is autonomous, but being under supervision and with periodic revisions. Martha decides to take care of him herself, due to emotional and economic reasons, but she lives $100 \mathrm{~km}$. away from her father. She suddenly faces a completely new situation: she tries to visit her father three times per week and contact him daily by phone to check how he is doing. She checks for any symptom to evaluate his status: how he is eating, how he is washing, if he looks dozy or disoriented, if the home is tidy, etc. After a month, Martha starts feeling a little bit stressed; she spent less time with her family and she has no time to meet her friends. Her husband and children demand more attention from her as well, and the situation is starting to overwhelm her. Martha wishes to have a system that supports her by monitoring her father remotely. With this system Martha could check the status of her father within 10 minutes every day reducing her travels only to the required ones when a problem is detected, previous confirmation via telephone. This would save her a lot of time on transportation and she could pay much more attention to her children, her work and her friends. 
TABLE I. ADLS

\begin{tabular}{|c|l|}
\hline General ADLs & $\begin{array}{l}\text { Sleeping, Waking-up, Dressing, Eating, } \\
\text { Resting, Bathing ... }\end{array}$ \\
\hline $\begin{array}{c}\text { Instrumental } \\
\text { ADLs }\end{array}$ & $\begin{array}{l}\text { Talking at the phone, Cooking, Watching TV } \\
\ldots\end{array}$ \\
\hline $\begin{array}{c}\text { ADLs on this } \\
\text { work }\end{array}$ & $\begin{array}{l}\text { Sleeping, Waking-up, Toileting, Showering, } \\
\text { Eating, Leaving apartment }\end{array}$ \\
\hline
\end{tabular}

Peter is a 35 -year-old caregiver working for about 8 years in an ambulatory care service. He has to take care of around 20 persons in the region. He visits them on an average of twice a week. He provides the services his customers booked and controls that their status is all right. He performs also a regular evaluation, consisting of questionnaires to the person and closest relatives or friends and a short interview. Sometimes he discovers that some information is missing, as some persons forget to tell him, but also because some reject having any kind of problem. This leads to an incomplete evaluation where Peter cannot get all that he expects. There are also quality problems when referring to the caring documentation: normally the documents remain with the patient, thus, other caregivers have difficulties to access them and sometimes they even get lost [6]. The social services are thinking about increasing the elderly persons to 25 per caregiver due to the huge demand, so they are planning to reduce the dedicated time to every patient. Peter wishes to have a system that helps him to monitor the persons avoiding the time spent on his visits so he could attend more people in a more efficient way. With such a system it would be possible to perform or complement the evaluations in order to get more reliable information.

\section{B. Research Problem}

Activity recognition on AAL, or ADLs recognition, is currently a very active field. We can distinguish two main recognition techniques: specification-based and learning algorithms. A detail description is out of the scope and could be found by [7].

Besides the different techniques and their performance, current works are focused mainly on the detection of basic ADLs . ADLs themselves provide insufficient information to have an overview of the health status and they do no contribute directly to the monitoring. By detecting toileting, preparing meal, dressing, etc, like in [8,9], it is not possible to evaluate the health status of a person. That is why we want to go a step further and focus on the detection of more complex situations that would be relevant for care monitoring. ADLs are only the basic information to infer situations of interest for caregivers: the assisted person does not eat enough, the person is not doing personal hygiene himself in recent days, and so on.

Moreover, in the current work there are several aspects that do not contribute to the concept of real scenarios, which are the desirable evaluation fields: artificial environments, such as special equipped houses or living labs [8], short test durations, like a few hours or days, and even with subjects out of the target group. These evaluations are enough for the very first tests, but lack of relevance regarding the real world: a person will not feel like at his own home and, probably, he will not act likewise either, and a 30-year-old person behaves quite different as a 60 -year-old one.

We want to determine if the recognition of such situations in real scenarios, and which concrete situations, would be helpful for a home monitoring system addressed for formal and informal caregivers. Informal caregivers would then reduce the burden of taking care of a person, and formal caregivers could cope with more persons needing care and alleviate their workload. Thus, a monitoring system could allow persons to care for their relatives while keeping them at their home, which is the desire of most of the elders, and saving long distances. The system aims to provide also a safety feeling for both sides, the elders living at home, and the relatives that can monitor them. For the formal caregivers the system could provide an additional benefit, facilitating the tasks of doing reliable assessments and avoiding unnecessary travels, by having the health status of the person monitored.

\section{Methodological ApPROACH}

Our system aims to detect and recognize situations of interest. Situation is an abstract term with a broad meaning, we defined it in our context as facts based on the alteration, decrease or absence of normal ADLs performing, which bring relevant information about the health status of a person, like could be hygiene or nutrition problems. These situations can be seen as a further step from the merely ADLs.

Such situations and everyday problems are described extensively on nursing diagnoses [10]. We have established the premise of using a set of ambient and non-intrusive sensors as simple as possible, in order to obtain a major acceptance of the system: sensors such cameras or microphones could disturb the persons privacy; while wearable sensors could be forgotten or just rejected to be worn. We have taken the nursing diagnoses as a basis, analyzed the different areas and considered which situations are more relevant and still feasible to detect with the sensor set. The situations are matched to a specific location and to concrete sensors in order to be detected. The result is a set of situations classified in five main groups: hygiene, nutrition, mobility, sleeping and cognition. For a detailed classification of all the situations, see Table II.

These situations and set of sensors are then the basis of our monitoring system, which we explain in detail on the next section.

TABLE II. SitUATIONS ClASSIFICATION

\begin{tabular}{|l|l|}
\hline \multirow{2}{*}{ Hygiene } & Inadequate toileting \\
\cline { 2 - 2 } & Inadequate personal hygiene \\
\hline Nutrition & Deficient nutrition \\
\hline \multirow{2}{*}{ Mobility } & Inactivity \\
\cline { 2 - 2 } & Too much time in a room \\
\cline { 2 - 2 } & Inappropiate time in a room \\
\hline \multirow{4}{*}{ Sleeping } & Too oft \\
\cline { 2 - 2 } & Too short \\
\cline { 2 - 2 } & Too long \\
\cline { 2 - 2 } & Inappropiate time \\
\hline \multirow{2}{*}{ Cognition } & Door opened forgotten when leaving \\
\cline { 2 - 2 } & Leaving at inappropiate time \\
\hline
\end{tabular}




\section{MONITORING SYSTEM}

The system is composed of a context-aware middleware connected with ambient sensors, which is responsible for detecting the situations of interest from the sensor data, and a web application for the remote monitoring.

We have already implemented a first version of the system. This consists of two main components: the contextaware middleware openAAL [11], extended by us with the recognition algorithms, and a server with a remote monitoring web application. The middleware is responsible for detecting activities and situations from the sensor and context data stored. When the system detects one of the situations defined in the previous section, a warning with detailed information is generated and sent to the monitoring application for the caregiver. Let us take a deeper look at the system components.

openAAL is an OSGi and ontology based middleware. It is a context-management framework that provides an easy integration of AAL services. For a detailed description of the framework, see [11]. The framework supports the integration of rules called upfliters. We have defined an hierarchical ontology for the data model, distinguishing among different leves of abstraction for the information: from the lowest-level which is the sensor information to more abstract levels as could be the location of a person or the ADLs. This ontology provides more flexibility for inferring context information in general, and in this case specifically for our rule system described next.

In a first version of the system we have decided to implement specification or rule-based algorithms for the activity and situation recognition based on the following arguments: (1) these techniques are computationally simpler, (2) we avoid the training phase from learning algorithms, which could become a nuisance for a large amount of real households and (3) the flexibility these techniques bring us as explained next. We have defined and implemented a rule system to infer the different abstraction level information from the context data. Thus, the very basic rules abstract the sensor data information to a higher level information, and so on recursively. This allows us to have a more flexible system where any rule can be changed, modified or removed without affecting the implementation of the rest.

To illustrate how the rules work on the different information levels we consider the example of detecting hygiene problems: from the very low level, the basic rules abstract the motion sensor information, obtaining the person's location, e.g. person is in the bathroom. On the next level, using the location and the humidity and temperature sensor data, the system recognizes when the person takes a shower or washes himself. One level above, the system detects, based on the uplifted information and defined context information, if punctual hygiene problems arise, e.g. person did not shower or wash today. Finally, at a very high level, the rules infer if the punctual problems recur enough to consider the problem relevant to report the warning to the caregivers, e.g. the person did not wash himself in the last 3 days. The process is a combination of rules, where the output is used concurrently by other rules, abstracting more and more the information until desired information is achieved. As the rules only depend on concrete context information, they only rely on the output of other rules and not on the implementation, providing a flexibility grade to modify and improve the rules without direct repercussion on the others.

The remote monitoring application is a care management software (CareCM), where a monitoring module has been implemented. The application is based on a server, where the warning information is received from the middleware, and then it is detailed presented on the web interface. There are several features on this software to support the caregivers, like concrete details for each warning type (e.g. sleeping phase when sleeping problems), the option to set the status of the warning (open, on process, closed), the possibility to set a responsible for handling the warning, in case of professionals, and a free text block for receiving input from the users or writing notes.

There is a second warning output via mobile SMS. This is only sent in concrete cases that are acuter and need immediate response like inactivity or forgetting to close the door when leaving the home. The SMS is sent to the carer, but the information is also stored on the web application for better management, keeping it as the central system.

\section{Current Evaluation}

The evaluation is being carried out in real households. Our target subjects are people over 65 year old living alone. Currently we have done the installation in eight apartments in an assisted living facility, but we are working on expanding it to achieve more housholds for a relevant evaluation.

The duration of the evaluation will be at least 6 months. Due to the good network we have, we aim to have a stable testfield with the basic system for long-term, being able to add more functionality for evaluating new features. From the evaluation point of view, we have two main purposes to consider:

\section{1) Situation and activity recognition:}

The accuracy of the activity and situations recognition is a priority point of interest; by collecting feedback as references, we want to achieve satisfactory situations recognition. Therefore, we have two systems for collecting feedback: the web application for the caregivers itself, which has some fields for the user to confirm the validity or incorrectness of each warning. And secondly, there is a diary, in paper and in electronic format in a tablet, where the person annotates what he has done during the day: meals, laundry, leave the home, receive visits, etcetera. The subjects themselves are also able to confirm or refute the warnings detected, as they will receive them also on the tablet application. With this feedback we aim to evaluate the accuracy by true positives, false positives or false negatives. This process will iterate, thus, as soon as we receive an error from the situation detection, we will work on modifying the rules pursuing an improved recognition.

\section{2) Support for caregivers:}

On the other hand we want to evaluate how helpful and useful the system is for caregivers. In case of the informal caregivers, we will realize periodic questionnaires and 
interviews related to time consumed during caring tasks, personal satisfaction with the system, and so on. There is also a burden index test [12] that we will perform before, during and after the evaluation in order to better understand the system improvements and advantages. Regarding the formal caregivers, questionnaires and interviews will also be performed regularly, but oriented to the professional duties: assessment, monitoring, time saving, distances saving, and etcetera.

\section{EXPECTED CONTRIBUTION}

This work is currently in progress. We have developed a first version of the system and installed it in several households. The evaluation is in its very beginning phase, and we are still looking for more subjects for the installation. It will be an active evaluation, thus, with constant modifications: adding new scenarios, improving the current ones and showing more detailed monitoring information, and so on, depending on the evaluation itself and the feedback we receive.

Although we have not achieved any relevant results yet, we aim to contribute to the research community with two different issues:

\section{1) Situations recognition:}

We aspire to provide not only a set of activities but also more complex situations relevant for health status monitoring able to be detected with a simple set of ambient sensors. We want to determine how accurate such a system is, detecting the situations. These situations will be evaluated in real homes with real elderly people over a long time period, as explained in the previous section. Together with the set of situations, algorithms able to detect such situations would be provided.

\section{2) Usefulnes for caregivers:}

Apart from the situation recognition, we aim to demonstrate if such a system relieves and support caregivers when caring elders. After the evaluation we will determine if such a system provides a benefit for the caregivers, both formal and informal. By the results we could conclude if informal caregivers reduce their stress situations, if they feel comfortable and safety with these kind of monitoring system. For the formal caregivers we want to proof if the system could alleviate them on their work, by performig more reliable assessment, reducing visits to the clients and paper work and providing a real-time health monitoring system as well.

\section{FUTURE WORK}

As we already mentioned, new or improved scenarios will appear during the evaluation. For instance, new situations that we realize, they would be useful, new sensors to provide more context information, or new algorithms for the situation detection, are considered. Despite the evaluation and the improvement of the system, there is a lot of work that could be done.

With such a recognition system, further applications could be developed. For instance, an assistance system with proactive services that interact directly with the person when a concrete situation is performed, or a recognition system connected directly to a personal emergency response system (PERS), so emergencies could be directly in an emergency center notified, instead via the web application and the SMS.

Another important issue in AAL is the social interaction of the elders. Our system does not aim to isolate the elders, but only to provide assistance on caring for them. These interaction could be always kept. We encourage also new technology solutions to promote interaction like social networks or video conference to complement the system, which are already arising.

Finally it is also important to remark the lack of comparision among different recognition techniques due their heterogeneity: different sensors used, different data format, different interfaces, etc. It would be really interesting to have such a testbed where different techniques could be evaluated.

\section{ACKNOWLEDGEMENT}

This work is sponsored by the German Federal Ministry of Education and Research (BMBF) in the context of the project easyCare.

\section{REFERENCES}

[1] B. Rosales, C. Kunze, R. Görlitz, N. Krämer, T. Vetter, M. Wieser "Easycare service platform, assistive systems and services to support family caregivers", $4^{\text {th }}$ AAL German Congress, Jan 2011

[2] J. P.Hirdes, T. F. Smith, T. Rabinowitz T et al., "The Resident Assessment Instrument - Mental Health (RAI-MH): inter-rater reliability and convergent validity", Journal of Behavioral Health Services and Research 29(4): pp. 419-432, 2002

[3] J. L. Hedlund and B. W. Viewig, "The Hamilton rating scale for depression: a comprehensive review", Journal of Operational Psychiatry vol. 10: pp. 149-165, 1979

[4] C. Collin, D. T. Wade, S. Davies and V. Horne, "The Barthel ADL index: a reliability study", Int Disability Study vol. 10: pp.61-63, 1988

[5] J. Pruitt and J. Grudin, "Personas: practice and theory", In Proc. DUX 2003, ACM Press (2003), New York, USA, 1-15

[6] B. Rosales, M. Mueller-Gorchs and C. Kunze, "Scenario-based design of an ICT platform for mobile information services in ambulatory care nursing", Connecting Health and Humans Proceedings of NI2009 - The 10th Int Congress on Nursing Informatics, 2009

[7] J. Ye, S. Dobson and S. McKeever, "Situation identification techniques inpervasive computing: A review", Pervasive and Mobile Computing, 2011

[8] T. Kleinberger, A. Jedlitschka, H. Strof, S. Steinbach-Nordmann, and S. Prückner, "Evaluation of ADL detection in the EMERGE project",. in $3^{\text {rd }}$ German AAL Congress, Berlin Jan 2010

[9] T. Duong, H. Bui, D. Phung, and S. Venkatesh, "Activity recognition and abnormality detection with the switching hidden semi-Markov model", In Proc. IEEE Computer Vision and Pattern Recognition (CVPR-05), pages 838-845, 2005.

[10] T. H. Herdman, "Nursing diagnoses: definitions and classification 2009-2011,"Wiley-Blackwell: Singapore. 2009.

[11] P. Wolf, A. Schmidt, J. P. Otte, M. Klein, S. Rollwage, B. König-Ries "openAAL - the open source middleware for ambient-assisted living (AAL)", 2010 AALIANCE conference, pp. 1-5, 2010.

[12] S. H. Zarit, K. E. Reever and J. Bach-Peterson, "Relatives of the impaired elderly: correlates of feelings of burden", Gerontologist 1980, 20(6):649-55. 